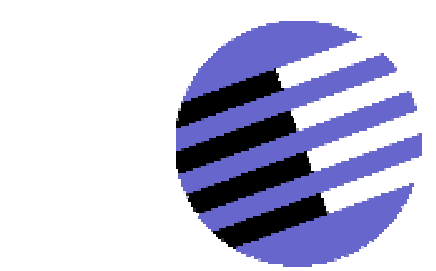

GOUERNANCE AND THE EFFICIENCY

OF ECONOMIC SYSTEMS

G E S Y

Discussion Paper No. 261
Signal-Jamming in a Sequential
Auction
Wei Ding*
Thomas D. Jeitschko**
Elmar Wolfstetter***

*Bonn Graduate School of Economics (BGSE), University of Bonn, Kaiserstr. 1, 53113 Bonn, Germany, Email: ding@uni-bonn.de.

${ }^{* *}$ Department of Economics, Michigan State University, East Lansing, MI 48824, USA, Email: jeitschk@msu.edu

${ }^{* * *}$ Corresponding Author: Department of Economics, Institute of Economic Theory I, Humboldt University at Berlin, Spandauer Str. 1, 10178 Berlin, Germany, Email: wolfstetter@gmail.com

\title{
June 2009
}

Financial support from the Deutsche Forschungsgemeinschaft through SFB/TR 15 is gratefully acknowledged. 


\title{
Signal-Jamming in a Sequential Auction*
}

\author{
Wei Ding \\ Bonn Graduate School of Economics \\ University of Bonn ${ }^{\dagger}$
}

\author{
Thomas D. Jeitschko \\ Michigan State University ${ }^{\ddagger}$ and \\ Royal Holloway, University of London
}

\author{
Elmar Wolfstetter \\ Institute of Economic Theory I \\ Humboldt-University at Berlin ${ }^{\S}$
}

April 2009

\begin{abstract}
In a recurring auction early bids may reveal bidders' types, which in turn affects bidding in later auctions. Bidders take this into account and may bid in a way that conceals their private information until the last auction is played. The present paper analyzes the equilibrium of a sequence of firstprice auctions assuming bidders have stable private values. We show that signal-jamming occurs and explore the dynamics of equilibrium prices.
\end{abstract}

JEL classifications: D44, D02, D43

Keywords: Auctions, Signaling, Price Competition

\footnotetext{
${ }^{*}$ We thank Yaron Raviv, Yosuke Yasuda, conference participants at the 2009 IIOC, and seminar participants at Korea University. Research support by the Deutsche Forschungsgemeinschaft (DFG), SFB Transregio 15, "Governance and Efficiency of Economic Systems," is gratefully acknowledged.

${ }^{\dagger}$ Bonn Graduate School of Economics (BGSE), University of Bonn, Kaiserstr. 1, 53113 Bonn, Germany, Email: ding@uni-bonn.de

${ }^{\star}$ Department of Economics, Michigan State University, East Lansing, MI 48824, USA, Email: jeitschk@msu.edu

${ }^{\S}$ Corresponding Author: Department of Economics, Institute of Economic Theory I, Humboldt University at Berlin, Spandauer Str. 1, 10178 Berlin, Germany, Email: wolfstetter@gmail.com
} 


\section{Introduction}

Many market transactions have an auction structure, and many such auctions are recurring events. For example, price competition between retailers is essentially a (reverse) auction. And this auction is typically a recurring event in which the relevant valuations (unit costs) are stable, at least for some time. Evidently, in this case bidders must pay attention to the information they reveal about their valuations through their bids. This gives rise to a problem of strategic information transmission.

The present paper analyzes equilibrium bidding in a sequence of first-price auctions, when bidders have stable private values. Bidders want to win each auction, but they are also concerned with concealing their valuation in order to reduce the intensity of price competition.

If bidders play strictly monotone strategies in the first auction, they reveal their private information, and the second auction is one under complete information, resulting in fierce competition that wipes out profits. Bidders thus attempt to keep their rival unsure about their valuation by playing non-monotone strategies, mimicking the bidder with a low valuation with some probability.

\section{The model}

Consider a sequence of two first-price auctions for two identical objects, and two ex ante symmetric bidders, named 1 and 2. Bidders draw their private valuation before the first auction and keep that valuation in second one. Before the second object is auctioned, bidders observe both bids of the first auction, and use this information to update their beliefs concerning their rival's valuation. Valuations $V$ are iid random variables which assume either a low value 0 (normalized) or a high value $v>0$ with the prior probability $\rho:=\operatorname{Pr}\{V=v\} \in(0,1)$.

If bidders tie, the winner is selected by flipping a fair coin, with one exception: If bidders tie in the second auction and exactly one bid was positive in the first auction, the one who made a positive bid in the first auction is selected as the winner.

We denote bidder $i$ 's bid in the $j$-th auction by $b_{i}^{j}$, and continuation payoffs by $\pi(h)$, where $h$ denotes the history of the game prior to the second auction.

The possible histories of the game, $h$, are described by past bids observed by both players. The following histories must be distinguished; there, only the sign of observed bids matters: 1) The history at the beginning of the game, $h_{\emptyset}$; 2) the histories with both bids either zero or positive: $h_{00}:=\left\{b_{1}^{1}=0, b_{2}^{1}=0\right\}$ and $h_{11}:=$ $\left.\left\{b_{1}^{1}>0, b_{2}^{1}>0\right\} ; 3\right)$ the histories with one positive bid and one bid equal to zero: $h_{10}:=\left\{b_{1}^{1}>0, b_{2}^{1}=0\right\}$ and $h_{01}:=\left\{b_{1}^{1}=0, b_{2}^{1}>0\right\}$. 


\section{Equilibrium}

A bidder with valuation $V=0$ obviously bids zero with certainty in both auctions (which will not be repeated from here onwards). This does not, however, imply that a zero bid can only come from a bidder with valuation $V=0$. Indeed, in a signaljamming equilibrium a bidder with $V=v$ may also bid zero in the first auction in order to keep his rival in doubt about his valuation.

We now solve the equilibrium strategies of a bidder with valuation $V=v$ in both auctions, for all possible histories of the game, employing the equilibrium concept of a sequential equilibrium with observable moves.

As a working hypothesis, suppose $F:[0, \bar{b}] \rightarrow[0,1]$ is the unique symmetric equilibrium mixed strategy of a bidder with $V=v$ in the first auction (history $h_{\emptyset}$ ), where $F(0)$ may be positive. This allows us to characterize the equilibrium play in the second auction, and then confirm the working hypothesis concerning the equilibrium play in the first auction. To avoid unnecessary duplication we state only the equilibrium strategies and beliefs of one player, named player 1.

Equilibrium in the second auction After the first auction, bidders observe bids, process this information to update their beliefs about the rival's valuation, and then play the second auction. Updated beliefs must be consistent with the equilibrium strategy of the first auction and the observed bids. Hence, using Bayes' rule whenever applicable, posterior beliefs are: ${ }^{1}$

$$
\operatorname{Pr}\left\{V_{2}=v \mid b_{2}^{1}\right\}=\left\{\begin{array}{lll}
1 & \text { if } & b_{2}^{1}>0 \\
\frac{F(0) \rho}{F(0) \rho+(1-\rho)}=: q & \text { if } & b_{2}^{1}=0 .
\end{array}\right.
$$

Proposition 1 (Second auction). Consider the second auction. The equilibrium strategy of player 1 (with $V_{1}=v$ ) depends on the history as follows: $h_{11} \Rightarrow b_{1}^{2}=v$, $h \in\left\{h_{00}, h_{01}\right\} \Rightarrow G\left(b_{1}^{2}\right), h=h_{10} \Rightarrow H\left(b_{1}^{2}\right)$ :

$$
\begin{aligned}
& G:[0, q v] \rightarrow[0,1], \quad G(b)=\frac{b(1-q)}{(v-b) q}=\frac{b(1-\rho)}{(v-b) \rho F(0)} \\
& H:[0, q v] \rightarrow[0,1], \quad H(b)=\frac{v(1-q)}{v-b}=\frac{v(1-\rho)}{(v-b)(1-\rho+\rho F(0))},
\end{aligned}
$$

where $H$ has one mass point at $b=0$. The associated equilibrium continuation payoffs are $\pi\left(h_{11}\right)=0, \pi\left(h_{00}\right)=\pi\left(h_{10}\right)=\pi\left(h_{01}\right)=v(1-q)$.

Proof. Equilibrium strategies and payoffs are self-evident for $h_{11}$. Histories $h_{01}$ and $h_{10}$ result in an asymmetric, and history $h_{00}$ in a symmetric one-shot auction, solved in Jeitschko and Wolfstetter (2002, Prop. 3).

\footnotetext{
${ }^{1}$ Note, this belief system involves only a fairly innocent prescription of "off-equilibrium path" beliefs by stipulating that $\operatorname{Pr}\left\{V_{2}=v \mid b_{2}^{1}>0\right\}=1$ also for bids that are higher than "predicted", i.e. for $b_{2}^{1}>\bar{b}$.
} 
Equilibrium in the first auction Signal jamming pays for bidder 1 only if bidder 2 has also a high valuation. However, signal jamming is costly, and its benefit outweighs the cost only if it is sufficiently likely that bidder 1 meets a high value bidder 2 .

Proposition 2. Suppose $\rho>1 / 3$. The equilibrium strategy in the first auction (conditional on $V=v)$ is $F:[0, \bar{b}] \rightarrow[0,1]:$

$$
\begin{gathered}
F(b):=\frac{1-\rho}{\rho} \frac{b}{v-b}+\frac{v}{v-b} \frac{\sqrt{3-4 \rho+\rho^{2}}-2(1-\rho)}{\rho} \\
\bar{b}:=v\left(2-\rho-\sqrt{3-4 \rho+\rho^{2}}\right) .
\end{gathered}
$$

$F$ has a mass point at zero: $F(0)=\sqrt{(1 / \rho-1)(3 / \rho-1)}-2(1 / \rho-1)>0$ which has a maximum at $\rho=3 / 4$ and approaches zero as $\rho \rightarrow 1$ and as $\rho \rightarrow 1 / 3$.

Proof. Consider one bidder, say bidder 1 with $V=v$, and history $h_{\emptyset}$ (first auction). To confirm the asserted equilibrium mixed strategy $F$, stated in (4), we must show that this bidder is indifferent between all bids from the support of $F$, which is $[0, \bar{b}]$ where $\bar{b}$ is stated in (5).

If bidder 1 with $V=v$ makes a bid $b \in(0, \bar{b}]$ his payoff is equal to

$$
\left(v-b+\pi\left(h_{11}\right)\right) \rho(F(b)-F(0))+\left(v-b+\pi\left(h_{10}\right)\right)((1-\rho)+\rho F(0)) .
$$

Whereas if he bids zero, his payoff is

$$
\left(\frac{v}{2}+\pi\left(h_{00}\right)\right)(\rho F(0)+(1-\rho))+\pi\left(h_{01}\right) \rho(1-F(0)) .
$$

And the assertion follows immediately.

We mention that no signal jamming occurs if $\rho \leq 1 / 3$; in that case, a high value bidder plays the myopic strategy $K:[0, \rho v] \rightarrow[0,1], K(b):=\frac{1-\rho}{\rho} \frac{b}{v-b}$ in the first auction, and in the second auction bids $v$ if he has observed a positive first-auction bid from his rival, and otherwise bid zero. While this is the unique Bayesian Nash equilibrium, that equilibrium is not a sequential equilibrium. ${ }^{2}$

\section{Signal-jamming}

Signal-jamming occurs if a bidder with a high valuation bids zero in the first auction with positive probability, $F(0)>0$, and thus sometimes mimics a bidder with a low valuation in order to keep the rival uninformed.

\footnotetext{
${ }^{2}$ For, suppose $V_{1}=v$ and consider history $h_{00}$; consistency of beliefs requires that each bidder believes that his rival has a zero valuation; but then bidding zero is not the best response of bidder 1 (in fact, no best response exists in that case).
} 
Altogether, bidder 1 benefits from signal jamming if and only if bidder 2 also has a high valuation. Signal jamming leads bidder 2 to update his belief from $\rho$ to $\operatorname{Pr}\left\{V_{1}=v \mid b_{1}^{1}=0\right\}=q<\rho$ instead of revealing bidder 1's type, which in turn induces him to bid stochastically lower, no matter how he bid in the first auction. If bidder 2 made a positive bid in the first auction, he plays the stochastically lower mixed strategy $H(b)>G(b), \forall b \in[0, q v]$, and if he also engaged in signal jamming, both bidders play the mixed strategy $G$ which preserves a positive expected profit in the second auction.

However, signal jamming is also costly since it entails the risk of losing the first auction. It follows that it pays to "invest" in signal-jamming only if it is sufficiently likely that the rival has a high valuation. Interestingly, this relationship is not monotone, and $F(0)$ has a global maximum at $\rho=3 / 4$.

We mention that signal-jamming induces pointwise less aggressive bidding in the first auction, in the sense that the myopic strategy $K$ first-order stochastically dominates the (continuously extended) strategy $\bar{F}(b):=\max \{F(b), 1\}$, i.e., $\bar{F}(b) \geq$ $K(b), \forall b \in[0, \rho v]$.

\section{Dynamics of equilibrium prices}

The study of price sequences in sequential auctions has received much attention in the literature (see, for example, McAfee and Vincent, 1993, Gale and Hausch, 1994, Jeitschko, 1999). In the present context, one might expect that prices be stochastically increasing since signal jamming involves bidding low in the first auction.

In the following assume $\rho>1 / 3$ (because otherwise no signal jamming occurs and equilibrium prices are stationary), and denote the continuously extended strategies for the enlarged domain $[0, v]$ by $\bar{G}(b):=\min \{G(b), 1\}, \bar{H}(b):=\min \{H(b), 1\}$.

Lemma 1. The probability distribution of the equilibrium price in the first auction, $F_{P^{1}}:[0, v] \rightarrow[0,1]$, is

$$
\begin{aligned}
F_{P^{1}}(p): & =\operatorname{Pr}\left\{P^{1} \leq p\right\} \\
& =\operatorname{Pr}\left\{\tilde{b}_{1}^{1} \leq p \quad \text { and } \tilde{b}_{2}^{1} \leq p\right\} \\
& = \begin{cases}(1-\rho+\rho F(p))^{2} & \text { if } p \leq \bar{b} \\
1 & \text { if } p \geq \bar{b}\end{cases}
\end{aligned}
$$

$F_{P^{1}}$ has exactly one mass point, $F_{P^{1}}(0)=\left((1-\rho)-\sqrt{3-4 \rho+\rho^{2}}\right)^{2}>0$, which is strictly decreasing in $\rho$ with $\lim _{\rho \rightarrow 1} F_{P^{1}}(0)=0$. 
Lemma 2. The probability distribution of the equilibrium price in the second auction, $F_{P^{2}}:[0, v] \rightarrow[0,1]$, is

$$
\begin{aligned}
F_{P^{2}}(p) & =\operatorname{Pr}\left\{P^{2} \leq p\right\} \\
& =\rho^{2}\left((1-F(0))^{2} \cdot 0+F(0)^{2} \bar{G}(p)^{2}+2 F(0)(1-F(0)) \bar{G}(p) \bar{H}(p)\right) \\
& +2 \rho(1-\rho)(F(0) \bar{G}(p)+(1-F(0)) \bar{H}(p))+(1-\rho)^{2} .
\end{aligned}
$$

$F_{P^{2}}$ has mass points at $p=0$ and $p=v$ :

$$
\begin{gathered}
F_{P^{2}}(0)=(1-\rho) \sqrt{3-4 \rho+\rho^{2}}>0 \\
\operatorname{Pr}\left\{P^{2}=v\right\}=\left(2-\rho-\sqrt{3-4 \rho+\rho^{2}}\right)^{2}>0 .
\end{gathered}
$$

$\operatorname{Pr}\left\{P^{2}=v\right\}$ is strictly increasing in $\rho$, and $F_{P^{2}}(0)$ decreasing with $\lim _{\rho \rightarrow 1} F_{P^{2}}(0)=$ 0 .

Proposition 3. Equilibrium prices are increasing in the sense of first-order stochastic dominance, $F_{P^{2}}(p) \leq F_{P^{1}}(p)$ (with strict inequality except for $p=v$ ), if and only if $\rho$ is sufficiently large, i.e. $\rho>\rho^{*}:=2-3 / 5 \sqrt{5}$.

This strong stochastic order does not apply to $\rho \in\left(1 / 3, \rho^{*}\right)$. However, in either case $E\left[P^{2}\right]>E\left[P^{1}\right]$.

Proof. 1) Suppose $\rho>\rho^{*}$. Let $p \in[0, v)$ and define (where the functions $G$ and $H$ are applied to the enlarged domain $[0, v)$ ):

$$
\begin{aligned}
\tilde{F}_{P^{2}}(p):= & \rho^{2}\left((1-F(0))^{2} \cdot 0+F(0)^{2} G(p)^{2}+2 F(0)(1-F(0)) G(p) H(p)\right) \\
& +2 \rho(1-\rho)(F(0) G(p)+(1-F(0)) H(p))+(1-\rho)^{2} .
\end{aligned}
$$

By definition, $G(p) \geq \bar{G}(p), H(p) \geq \bar{H}(p)$, with equality for all $p \in[0, q v]$ and strict inequality for all $p \in(q v, v)$. Therefore, it follows immediately that $\tilde{F}_{P^{2}}(p)$ is a pointwise upper bound of $F_{P^{2}}(p)$, i.e., $\tilde{F}_{P^{2}}(p) \geq F_{P^{2}}(p)$, for all $p \in[0, v)$, and hence, in particular, for all $p \in[0, \bar{b}]$.

As one can easily confirm, $F_{P^{1}}>\tilde{F}_{P^{2}}(p), \forall p \in[0, \bar{b}] \Longleftrightarrow \rho>\rho^{*}$. Since $F_{P^{1}}(p)=$ $1, \forall b \geq \bar{b}$ and $F_{P^{2}}<1, \forall b<v$ (since it has a mass point at $p=v$ ), we conclude that $\rho>\rho^{*} \Rightarrow F_{P^{2}}(p) \leq F_{P^{1}}(p)$, with strict inequality everywhere except at $p=$ $v$, as illustrated in the right-hand Figure 1. Of course, the established first-order stochastic dominance relationship implies $E\left[P^{2}\right]>E\left[P^{1}\right]$.

2) Suppose $\rho \in\left(1 / 3, \rho^{*}\right)$. Then, as one can easily confirm, $F_{P^{2}}(0)>F_{P^{1}}(0)$. Moreover, $F_{P^{1}}(p)=1>F_{P^{2}}(p), \forall p \in[\bar{b}, v)$ (since $F_{P^{1}}(\bar{b})=1$ and $F_{P^{2}}$ has a mass point at $p=v)$. Therefore, $F_{P^{2}}(p)$ and $F_{P^{1}}(p)$ must intersect at least once; hence no firstorder stochastic dominance relationship applies to $P^{2}$ and $P^{1}$, as illustrated in the left-hand Figure 1. 

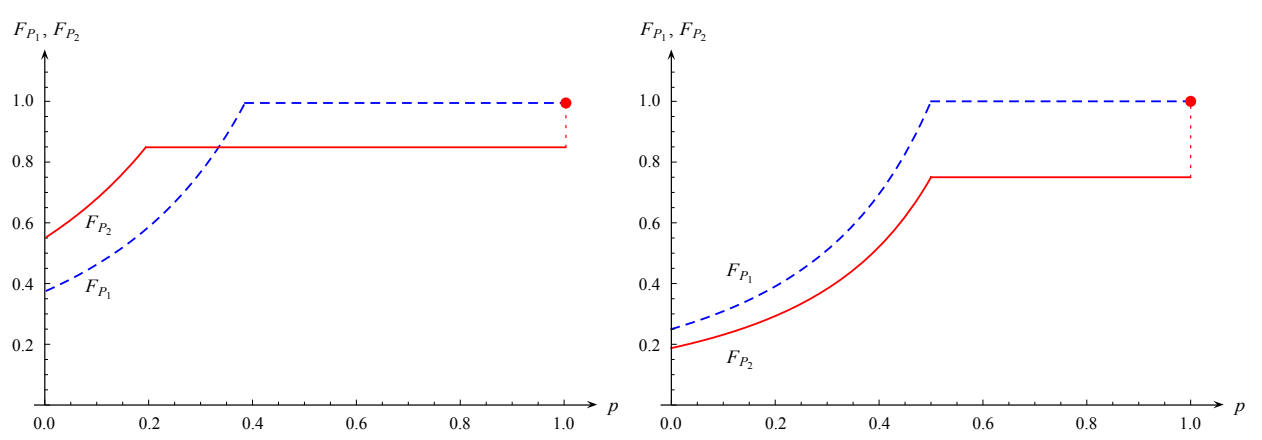

Figure 1: Comparison between $F_{P^{1}}$ (dashed) and $F_{P^{2}}$ (solid) for $v=1$ and $\rho=1 / 2<$ $\rho^{*}$ (left) resp. $\rho=3 / 4>\rho^{*}$ (right)

3) Computing expected equilibrium prices, one finds, using (8) and then (4), (5)),

$$
\begin{aligned}
E\left[P^{1}\right] & =\int_{0}^{v} p d F_{P^{1}}(p)=\int_{0}^{\bar{b}} 2 p(1-\rho+\rho F(p)) \rho d F(p) \\
& =v\left(7-2 \rho(4-\rho)-2(2-\rho) \sqrt{3-4 \rho+\rho^{2}}\right) .
\end{aligned}
$$

Similarly, the expected price in the second auction auction is, using equations (9) and (11),

$$
\begin{aligned}
E\left[P^{2}\right] & =\int_{0}^{v} p d F_{P^{2}}(p)=\int_{0}^{q v} p d F_{P^{2}}(p)+v \operatorname{Pr}\left\{P^{2}=v\right\} \\
& =\frac{v\left(14-5 \rho^{3}+25 \rho^{2}-34 \rho-\left(8+5 \rho^{2}-14 \rho\right) \sqrt{3-4 \rho+\rho^{2}}\right)}{\sqrt{3-4 \rho+\rho^{2}}-(1-\rho)}
\end{aligned}
$$

Therefore, after some rearrangements, for all $\rho>1 / 3$ :

$$
E\left[P^{1}\right]-E\left[P^{2}\right]=v \sqrt{3-4 \rho+\rho^{2}}\left(5 \rho-7+4 \sqrt{3-4 \rho+\rho^{2}}\right)<0,
$$

as asserted.

\section{Discussion}

In the present paper we assumed that bidders have stable valuations. An alternative framework would be to assume that valuations are subject to stochastic scale effects, as in Jeitschko and Wolfstetter (2002).

We also assumed that bidders observe all first auction bids before they bid in the second auction. If instead bidders could only learn whether they either won or lost the first auction, in some subgames bidders would know the rank order of valuations, as in Landsberger et al. (2001). 
Our analysis also assumed a passive auctioneer. Therefore, signal jamming served exclusively the purpose of misleading the rival bidder. The incentive for signal jamming is further increased if the auctioneer is able adjust reserve prices, taking advantage of information acquired during the first auction.

\section{References}

Gale, D., Hausch, D., 1994. Bottom-fishing and declining prices in sequential auctions. Games and Economic Behavior 7, 318-331.

Jeitschko, T., 1999. Equilibrium price paths in sequential auctions with stochastic supply. Economics Letters 64, 67-72.

Jeitschko, T., Wolfstetter, E., 2002. Scale economies and the dynamics of recurring auctions. Economic Inquiry 40, 403-414.

Landsberger, M., Rubinstein, J., Wolfstetter, E., Zamir, S., 2001. First-price auctions when the ranking of valuations is common knowledge. Review of Economic Design 6, 461-480.

McAfee, R. P., Vincent, D., 1993. The declining price anomaly. Journal of Economic Theory 60, 191-212. 